Research report

\title{
Evaluation of performance monitoring ERPs through difficulty manipulation in a response-feedback paradigm
}

\author{
Bertille Somon $^{\mathrm{a}, \mathrm{b}}$, , Aurélie Campagne ${ }^{\mathrm{b}}$, Arnaud Delorme ${ }^{\mathrm{c}, \mathrm{d}}$, Bruno Berberian ${ }^{\mathrm{a}}$ \\ a ONERA The French Aerospace Lab, Information Processing and Systems Department, 13661 Salon Cedex Air, France \\ ${ }^{\mathrm{b}}$ Univ. Grenoble Alpes, CNRS, LPNC, 38000 Grenoble, France \\ c Centre de Recherche Cerveau et Cognition, CNRS, Université Paul Sabatier, Toulouse, France \\ ${ }^{\mathrm{d}}$ Swartz Center for Computational Neuroscience, Institute of Neural Computation, University of California, San Diego, CA, USA
}

\section{A R T ICLE INFO}

\section{Keywords:}

Electroencephalography (EEG)

Error-related negativity (ERN)

Feedback-related negativity (FRN)

Flanker task

Surface Laplacian

\begin{abstract}
A B S T R A C T
Performance monitoring is an amply studied function, since it is of major importance in carrying out actions in our everyday life. No consensus has been reached on the functional role and the relationship between each event-related potential (ERP) characterizing this function. In this study, we used a modified version of the flanker task, measuring the impact of task difficulty on the amplitudes of response-locked and feedback-locked performance monitoring ERPs in a single trial. We observed a functional differentiation between fronto-central (ERN/ CRN and FRN) and centro-parietal (Pe/Pc and P300) components: the former seem to be only sensitive to accuracy, whereas the latter seem to be mainly modulated by task difficulty. The use of a surface Laplacian transformation, estimating current source density, on our data also supported an effect of difficulty on centro-parietal response-locked and feedback-locked ERPs. This technique allowed the spatial resolution to be improved and provided clarity, associated with the difficulty manipulation, on the activity of response-locked and feedback-locked performance monitoring ERPs.
\end{abstract}

\section{Introduction}

Performance monitoring represents one's ability to assess the accuracy of one's own or of others' actions. This area of study has drawn a lot of attention over the past decades, since this function plays a crucial role in our everyday life, e.g., when we learn (Holroyd and Coles, 2002; Luft, 2014). Effective action monitoring relies on the ability to detect errors and adjust performance in accordance with the situation at hand (Falkenstein et al., 2000). Performance monitoring has been studied at the behavioral level (Rabbitt, 1966a, 1966b), but also through neuroimaging techniques, like electroencephalography (EEG; Dehaene et al., 1994; Falkenstein et al., 1991; Gehring et al., 1993, 1990), functional magnetic resonance imaging (fMRI) (Iannaccone et al., 2015; Ullsperger et al., 2007; Van Veen and Carter, 2002) or magnetoencephalography (MEG) (Keil et al., 2010; Luck and Kappenman, 2011). In EEG, two now well-known event-related potentials (ERPs) time-locked to the participant's response were identified: (i) a negative

\footnotetext{
* Corresponding author at: ONERA, Base Aérienne 701, 13661 Salon Cedex Air, France.

Email address: bertille.somon@onera.fr (B. Somon)
}

ERP peaking at fronto-central sites around $80 \mathrm{~ms}$ after making a correct response (called "the correct-related negativity", or CRN) (Allain et al., 2004; Vidal et al., 2000), or an error (called "the error-related negativity", or ERN) (Falkenstein et al., 1991; Gehring et al., 1990); (ii) a following positive component that peaks at centro-parietal sites between 250 and $400 \mathrm{~ms}$ after making a correct response (called "the correct Positivity", or Pc) (Van der Borght et al., 2016) or an error (called "the error Positivity", or Pe) (Falkenstein et al., 1991; Overbeek et al., 2005). The ERN/CRN component has been argued to correspond to an evaluation process of the response that one has just performed. Conversely, two main theories have been put forward for the functional role of the $\mathrm{Pe} / \mathrm{Pc}$ component, with no consensus having been reached yet: (i) the $\mathrm{Pe} / \mathrm{Pc}$ could play a functional role in conscious error detection (Nieuwenhuis et al., 2001; Rigoni et al., 2015; Scheffers et al., 1996; Vidal et al., 2000), or (ii) this positivity could be a response-locked P300 specific to performance monitoring, thus its functional significance would be attention allocation (Falkenstein et al., 2000; O'Connell et al., 2007; Ridderinkhof et al., 2009). 
Performance monitoring is also characterized by ERPs time-locked to the display of feedback regarding performances. Two ERPs associated with feedback evaluation were identified in multiple studies. First, a negative component peaking at fronto-central sites $250 \mathrm{~ms}$ after a "worse-than-expected" or unanticipated feedback is given to the participant (called "the feedback-related negativity", or FRN) (Cavanagh and Frank, 2014; Luu et al., 2003; Miltner et al., 1997). Functional theories on the FRN largely argue that this ERP is a manifestation of the expectation of a stimulus (Holroyd et al., 2006; Holroyd and Coles, 2002; Oliveira et al., 2007). It is assumed that the FRN plays the role of reinforcement-learning or prediction of response outcome (PRO theory) (Alexander and Brown, 2011, 2010), through the evaluation of feedback stimulus expectation, whether it is linked to contingent action or not (Donkers and Van Boxtel, 2005; Holroyd and Coles, 2002; Yeung et al., 2004). Another recent functional theory suggests that the FRN would not be a negativity, but rather, would instead reflect a reward-related positivity (RewP) (Kujawa et al., 2013) that would be suppressed following a negative feedback/nonreward (Proudfit, 2015). The amplitude of this reward-related positivity, peaking at around 250-300 ms after feedback, would be modulated by both the veracity and percentage of the rewards in gambling or door tasks (see Sambrook and Goslin, 2015 for a meta-analysis on the effect of magnitude and feedback likelihood on the FRN/RewP). Finally, the FRN has been shown to interrupt a positive wave that is maximal at centro-parietal sites between 250 and $400 \mathrm{~ms}$ after the feedback display: the P300 (Sato et al., 2005; Yeung et al., 2004). Actually, the role of the $\mathrm{P} 300$ as a feedback-locked potential is assumed to be the same as its usual functional significance, i.e., information processing or attention allocation (Scheffers and Coles, 2000).

Although the performance monitoring system is being amply studied $^{1}$, a few keys are still missing, such as the relationship between response-locked and feedback-locked potentials, which remains under debate. Most of the performance monitoring ERPs have generally been studied separately, in order to define their functional relevance, and only a few studies have explored their link by using single trials in which both response from the participant and feedback were provided (e.g., Gentsch et al., 2009; Luu et al., 2003; Müller et al., 2005). Gentsch et al. (2009) studied correct, error and unexpected error feedback, but didn't provide any stimulus for error feedback, increasing ambiguity in the data. Luu et al. (2003) on their part, only looked at the effect of various rewards on correct feedback.

Interestingly, recent studies have proposed that perceptual or cognitive load be modulated in stimulus-response tasks, in order to have a better understanding of ERP functions. As an illustration, Van der Borght et al. (2016) manipulated levels of difficulty in a performance monitoring task and studied their effect on response-locked ERPs. They showed that ERN/CRN and Pe/Pc components were modulated by the difficulty level of the task. For the ERN/CRN components, a difficulty $x$ accuracy interaction revealed an increase in the ERN minus CRN amplitude difference in the easy condition. On the other hand, the Pe/ Pc component globally increased in the easy condition. These authors and others argued that these effects depend on the difficulty level (as reflected by the error rate) and/or the methodology used to modulate the task difficulty (Endrass et al., 2012; Grützmann et al., 2014). In addition, task difficulty is acknowledged to also affect the impact of feedback on task performance at the behavioral level (Luft, 2014). However, the extent to which task difficulty modulates feedback-locked ERPs remains largely unknown. The aim of the present EEG study is thus to investigate the impact of task difficulty on neural components associated with performance monitoring during both response and

1 For reviews on performance monitoring components and their applications see Gehring et al. (2011) or Somon et al. (2017). feedback. For this purpose, participants performed a modified version of the flanker task, allowing the study of both response-related and feedback-related brain activities in a single trial. Seventeen right-handed, healthy participants were asked to respond as fast as possible according to the direction of a target arrow displayed for $10 \mathrm{~ms}$. The level of task difficulty was manipulated using distractors or not. In an easy condition,only the target arrow was displayed in the middle of the screen, whereas in a more difficult condition, the target arrow was flanked above and below by other arrows. The flankers were heading either in the same direction (congruent trial) or in the opposite direction (incongruent trial) to the target arrow. Participants were time-pressured to respond to the target. Feedback was given to the participant at the end of every trial. The electroencephalographic activity of the participants was recorded continuously. For each trial, EEG activity was analyzed both at the time of the response and at feedback onset, according to the accuracy of the participant and the level of difficulty.

Regarding the effect of accuracy on the response-locked ERPs, we expect an increase in the ERN and the Pe for errors, compared to the CRN and Pc associated with correct responses, in accordance with the literature (Gehring et al., 2011; Holroyd and Coles, 2002; Van der Borght et al., 2016). For feedback-locked ERPs, an increase in the FRN for error feedback compared to correct feedback is also assumed (Cavanagh and Frank, 2014; Luu et al., 2003; Miltner et al., 1997). In contrast, we expect no effect of accuracy on the P300 component following feedback. Indeed, several studies suggest no effect of the valence and magnitude of feedback on this component (Sato et al., 2005; Wu and Zhou, 2009; Sambrook and Goslin, 2015). Based on this literature, we assume that the information processing and attention allocated to negative and positive feedback should be similar.

Regarding the effect of difficulty on response-locked ERPs, several studies showed that the amplitudes of the ERN, CRN and Pe components were modulated by uncertainty and task difficulty (Van der Borght et al., 2016; Pailing and Segalowitz, 2004; Endrass et al., 2012). Based on the results of this literature, we expect a decrease in the amplitude of the ERN and Pe, coupled with an increase in the CRN amplitude in the difficult condition compared to an easy condition. Indeed, the degree of uncertainty in the difficult condition of our study is higher due to the visual complexity of the stimulus coupled with the time pressure. Consequently, we assume that the amplitude difference, both between the ERN (error) and CRN (correct response), and between the Pe (error) and Pc (correct response), will be reduced with increasing task difficulty. For feedback-locked ERPs, the FRN is assumed to be activated by unanticipated or worse-than-expected feedback, and it is accepted that a perceptually more difficult condition reduces the certainty of one's response and prediction abilities of response outcome. Consequently, we assume that the amplitude difference of the FRN between errors and correct responses will be reduced in the difficult condition compared to the easy condition. An increase in the P300 amplitude is also reported with increasing uncertainty (Scheffers and Coles, 2000) and would illustrate an increase in the relevance of feedback. We thus assume a higher P300 amplitude in the difficult condition than in the easy condition. An interesting avenue tackled by our study concerns the relative modulation of the different components. Regarding functional theories of these different ERPs, we expect that an increase in the Pe/Pc component will be associated with a decrease of the FRN and P300. Indeed, an increase in error salience leads feedback to be less surprising (decrease of the FRN), and less relevant (decrease of the P300). Likewise, we expect that an increase in the ERN/CRN will lead to a decrease of both the FRN and P300, since a better evaluation of the stimulus and of the response performed will lead to an expected feedback (smaller FRN) and less information obtained from this feedback stimulus (smaller P300).

Something noteworthy is that the study of the variation of the various components may be biased by the low spatial resolution of surface 
ERPs. Thus, in order to improve our ability to distinguish surface ERP variations, the event-related potentials time-locked to the response and feedback were analyzed by applying a particular signal-processing technique: the surface Laplacian Transformation. Interestingly, this technique first allowed Vidal et al. (2003) to show the existence of the CRN after correct responses. It has proven to be very efficient in dissociating and localizing ERP components associated with performance monitoring (Allain et al., 2004; Vidal et al., 2000). More generally, this technique improves spatial, as well as temporal, EEG resolution (Burle et al., 2015). Moreover, surface Laplacian is physiologically relevant (Roger et al., 2010; Van der Borght et al., 2016) as opposed to other component isolating analyzes, like independent or principal-component analysis (ICA or PCA), which are only statistical and can lead to results that are not physiologically plausible (Delorme et al., 2012). The use of this technique in the present study may improve the ERP analysis, since it limits the impact of components on each other, particularly for components such as the Pe or P300, which are very broad and diffusing ERPs that can therefore influence recordings quite far from their sources. Component isolating analyses are not meant to dissociate clearly relative influences of components from each other. The surface Laplacian transformation will allow us to better distinguish performance monitoring ERPs and also to learn more about their spatial localization and functional role, as previously reported (Burle et al., 2008; Carvalhaes and de Barros, 2015; Gevins, 1989; Tenke and Kayser, 2012; Van der Borght et al., 2016; Vidal et al., 2003).

\section{Results}

\subsection{Behavioral data}

Values for RTs and error rates are all reported in Table 1.

\subsubsection{Reaction times}

The mean reaction time (mRT) was significantly modulated by accuracy $\left(\mathrm{F}(1,16)=11.12, \quad \mathrm{p}<.005, \quad \eta^{2}{ }_{\mathrm{p}}=0.41\right) \quad$ and difficulty $\left(\mathrm{F}(2,32)=33.99, \mathrm{p}<.001, \varepsilon_{\mathrm{GG}}=0.997, \mathrm{\eta}_{\mathrm{p}}^{2}=0.68\right.$; see Table 1$)$. With regard to accuracy, mean comparisons showed that mRT was significantly shorter for error trials than for correct trials. With regard to difficulty, mean comparisons revealed a shorter mRT (i) in the easy condition compared to the difficult condition, congruent and incongruent (both ps $<0.001$ ), and (ii) in the difficult congruent condition compared to the difficult incongruent condition $(\mathrm{p}<.01)$.

\subsubsection{Error rates}

The mean error rate (mER) was significantly modulated by difficulty $\left(\mathrm{F}(2,32)=47.86, \mathrm{p}<.001, \varepsilon_{\mathrm{GG}}=0.589, \eta_{\mathrm{P}}^{2}=0.75\right.$, see Table 1$)$. Mean comparisons showed a lower mER in the easy condition than in the difficult incongruent condition $(\mathrm{p}<.001)$. The mER was also significantly lower in the difficult congruent condition than in the difficult incongruent condition $(\mathrm{p}<.001)$. No significant difference of mER was observed between the easy condition and the congruent difficult condition.

\subsection{Response-locked potentials}

\subsubsection{Monopolar recordings}

2.2.1.1. ERN/CRN The ERN/CRN amplitude was significantly modulated by accuracy $\left(\mathrm{F}(1,16)=68.76, \mathrm{p}<.001, \mathrm{\eta}_{\mathrm{p}}^{2}=0.81\right)$ and by the accuracy $x$ difficulty interaction $\left(\mathrm{F}(1,16)=8.99, \mathrm{p}<.01, \mathrm{\eta}_{\mathrm{p}}^{2}=0.36\right)$. Mean comparisons revealed that the ERN amplitude (error trials) was significantly higher than the CRN amplitude (correct trials) in both the easy and the difficult conditions (both ps $<0.001$ ). However, no significant amplitude difference was observed between the easy and the difficult conditions for both CRN and ERN amplitudes. The interaction effect was observable in the fact that the amplitude of the difference wave (ERN minus CRN; i.e., the ERP difference between error and correct trials) was significantly higher in the easy condition $(-8.10 \pm 1.00 \mu \mathrm{V})$ than in the difficult condition $(-5.26 \pm 0.86 \mu \mathrm{V}$ $\mathrm{t}(16)=-2.62, \mathrm{p}<.05)$. These results are observable in Fig. 1a.

\subsection{2. $\mathrm{Pe} / \mathrm{Pc}$}

The $\mathrm{Pe} / \mathrm{Pc}$ amplitude was significantly modulated by accuracy $\left(\mathrm{F}(1,16)=34.66, \mathrm{p}<.001, \eta_{\mathrm{p}}^{2}=0.68\right)$, difficulty $(\mathrm{F}(1,16)=6.30$, $\left.\mathrm{p}<.05, \quad \eta^{2}{ }_{\mathrm{p}}=0.28\right)$ and the accuracy $\mathrm{x}$ difficulty interaction $\left(\mathrm{F}(1,16)=11.40, \mathrm{p}<.005, \mathrm{\eta}_{\mathrm{p}}^{2}=0.42\right)$. Mean comparisons revealed that the Pe amplitude (error trials) was significantly higher than the Pc amplitude (correct trials) in both the easy and the difficult conditions $(\mathrm{p}<.001$ and $\mathrm{p}<.05$ respectively). The Pe amplitude was significantly reduced in the difficult condition compared to the easy condition $(\mathrm{p}<.05)$. However, no significant difference was observed between the easy and the difficult conditions for the Pc amplitude. The interaction effect was observable in the fact that the amplitude of the difference wave (Pe minus Pc; i.e., the ERP difference between error and correct trials) was significantly higher in the easy condition $(6.01 \pm 0.87 \mu \mathrm{V})$ than in the difficult condition $(3.01 \pm 0.90 \mu \mathrm{V} ; \mathrm{t}(16)=-3.38, \mathrm{p}<0.005)$. These results are observable in Fig. $1 \mathrm{~b}$.

\subsubsection{Surface-Laplacian-transformed ERPS}

2.2.3.1. ERN/CRN First of all, we can see in Fig. 1c that, compared to the results with monopolar recordings, the positive wave interrupting both the ERN and CRN disappears after a surface Laplacian is applied, as previously reported by Allain et al. (2004) and Van der Borght et al. (2016). We also observe a better definition of the ERN activity on scalp maps (see Fig. 3). The statistical analysis performed on the ERN/CRN current source density (CSD) showed a main effect of accuracy $\left(\mathrm{F}(1,16)=30.35, \mathrm{p}<.001, \mathrm{\eta}_{\mathrm{P}}^{2}=0.65\right)$. The CSD amplitude of the ERN was significantly higher than the CSD amplitude of the CRN. Like for monopolar recording, there was no main effect of task difficulty on the ERN/CRN CSD amplitude. Interestingly, the effect of the accuracy $x$ difficulty interaction disappeared with the application of the surface Laplacian $(\mathrm{F}(1,16)=2.72, \mathrm{p}=.12)$.

2.2.3.2. $P e / P c$ We can see on the scalp maps, that the surface Laplacian transformation again defined the potentials more clearly (see Fig. 3). The CSD amplitude of the Pe/Pc was again significantly modulated by accuracy $\left(F(1,16)=16.19, \mathrm{p}<.001, \eta_{\mathrm{P}}^{2}=0.50\right)$ and the accuracy $\mathrm{x}$ difficulty interaction $\left(\mathrm{F}(1,16)=9.31, \mathrm{p}<.01, \mathrm{n}_{\mathrm{p}}^{2}=0.37\right)$. The main effect of difficulty on Pe/Pc CSD amplitude has disappeared. Mean comparisons revealed that the CSD amplitude of the Pe (error tri-

Table 1

Behavioral results during execution of a modified version of the flanker task with the various difficulty levels

\begin{tabular}{|c|c|c|c|c|c|}
\hline & & All conditions & Easy & Difficult congruent & Difficult incongruent \\
\hline Mean & Error trials & $373 \pm 7$ & $337 \pm 10$ & $384 \pm 14$ & $397 \pm 8$ \\
\hline $\mathrm{RT}$ (ms) & Correct trials & $393 \pm 4$ & $367 \pm 6$ & $391 \pm 5$ & $420 \pm 6$ \\
\hline \multicolumn{2}{|c|}{ Mean ER (\%) } & $15.14 \pm 1.82$ & $4.94 \pm 0.97$ & $6.76 \pm 1.63$ & $33.70 \pm 4.14$ \\
\hline
\end{tabular}


a)

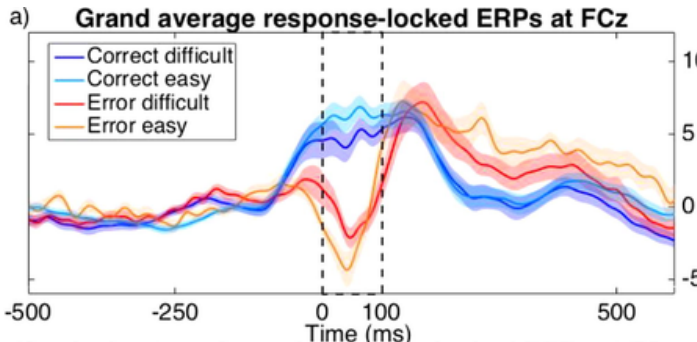

c)Laplacian transformed response-locked ERPs at FCZ

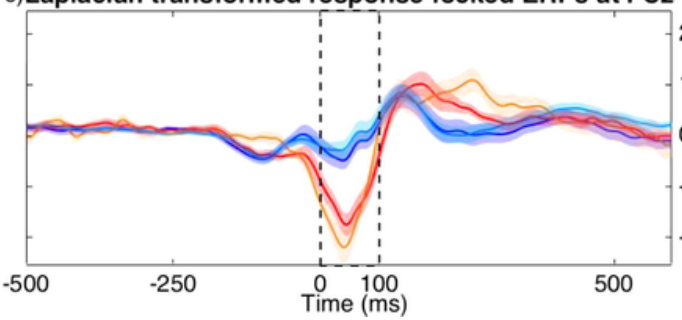

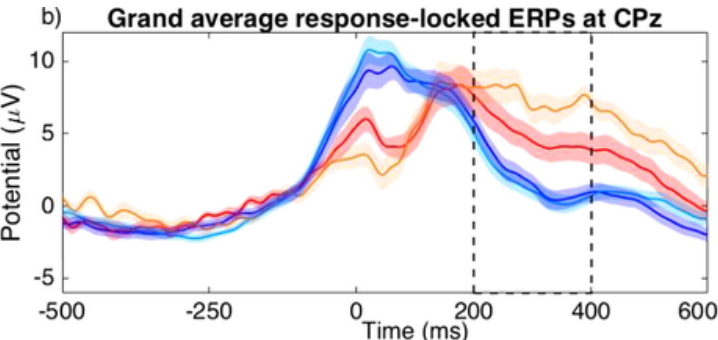

d)Laplacian transformed response-locked ERPs at CP

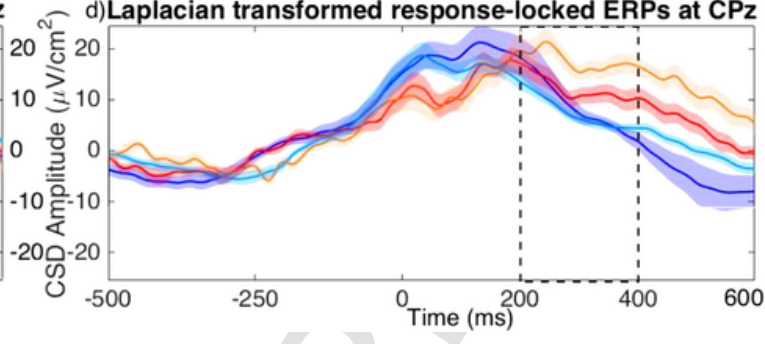

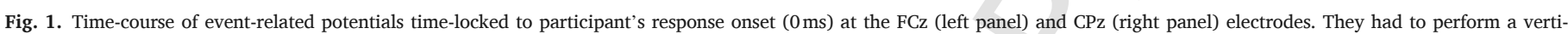

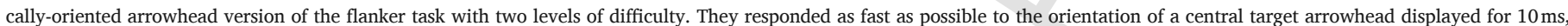

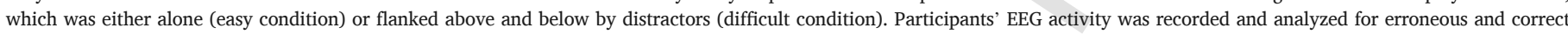

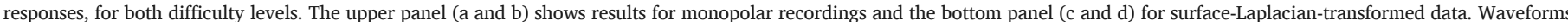

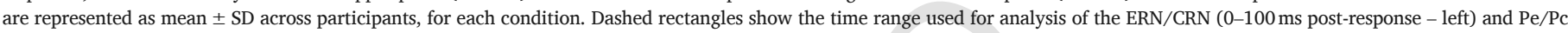
(200 - 400 ms post-response - right).

als) was significantly higher than the CSD amplitude of the Pc (correct trials) in the easy condition ( $\mathrm{p}<.001)$. There was no significant difference between the CSD amplitudes of Pe and Pc in the difficult condition. For error trials, but not for correct trials, the CSD amplitude was significantly increased in the easy condition compared to the difficult one $(\mathrm{p}<.05)$. In accordance with monopolar analyses, the interaction was shown to be relevant when comparing the difference wave (Pe minus Pc; i.e., the ERP difference between error and correct trials). We observed that the difference wave was significantly higher in the easy condition $\left(10.26 \pm 1.70 \mu \mathrm{V} . \mathrm{cm}^{-2}\right)$ than in the difficult condition $\left(3.17 \pm 2.32 \mu \mathrm{V} . \mathrm{cm}^{-2} ; \mathrm{t}(16)=-3.05, \mathrm{p}<0.01\right)$. These results are observable in Fig. 1d.
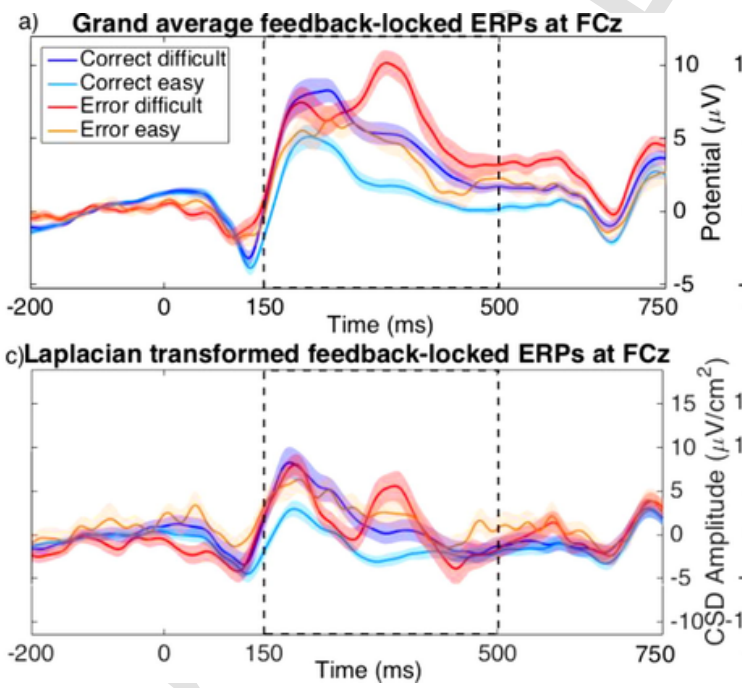

\subsection{Feedback-locked potentials}

\subsubsection{Monopolar recording}

2.3.1.1. FRN The FRN amplitude was only significantly modulated by accuracy $\left(\mathrm{F}(1,16)=8.66, \mathrm{p}<.01, \mathrm{\eta}_{\mathrm{P}}^{2}=0.35\right)$. It was significantly higher for error feedback than for correct feedback. These results are observable in Fig. 2a.

2.3.1.2. $P 300$ Conversely, the $\mathrm{P} 300$ amplitude was only significantly modulated by difficulty $\left(\mathrm{F}(1,16)=20.23, \mathrm{p}<.001, \eta_{\mathrm{P}}^{2}=0.56\right)$. It was significantly higher in the difficult condition than in the easy one.

These results are observable in Fig. $2 b$.

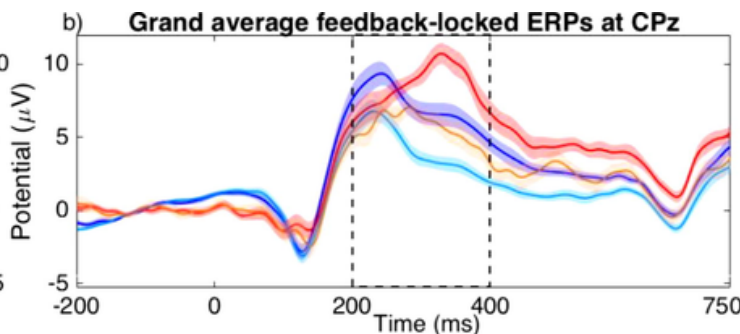

d) Laplacian transformed feedback-locked ERPs at CPz

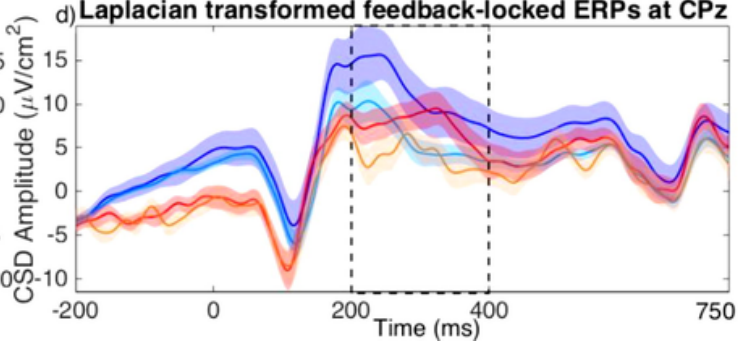

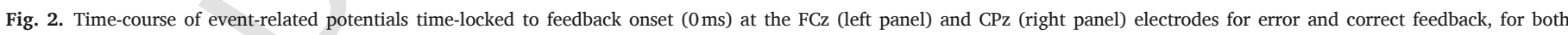

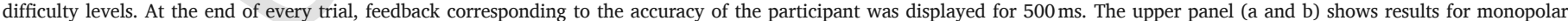

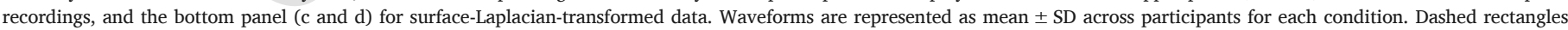

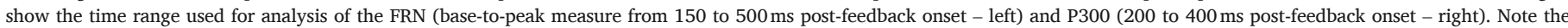

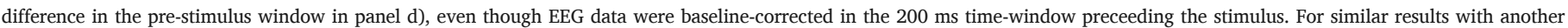
baseline correction, see Supplementary materials. 

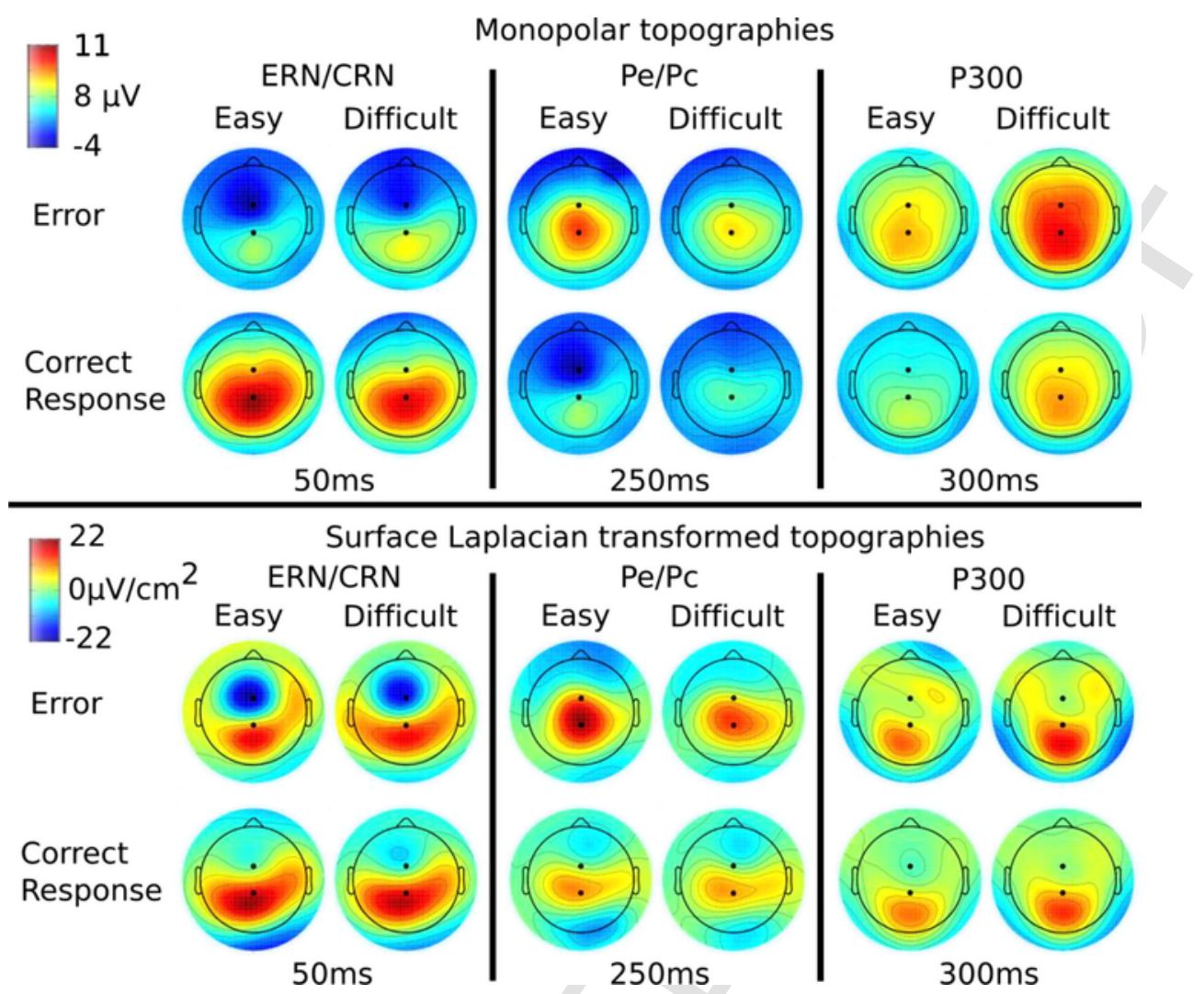

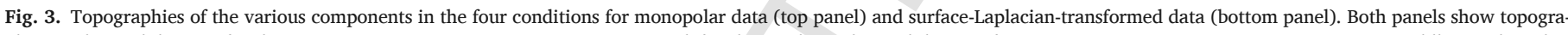

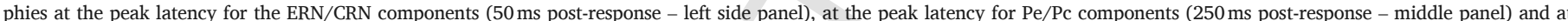

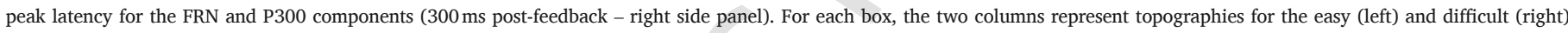

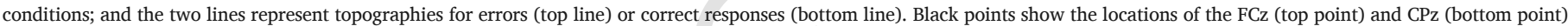
electrodes. Note the two different scales for monopolar (in $\mu \mathrm{V}$ ) and surface-Laplacian-transformed data (in $\mu \mathrm{V} / \mathrm{cm}^{2}$ ).

\subsubsection{Surface-Laplacian-transformed ERPS}

2.3.2.1. FRN In accordance with monopolar data, the CSD amplitude of the FRN was only modulated by accuracy $(\mathrm{F}(1,16)=32.54$, $\left.\mathrm{p}<.001, \eta_{\mathrm{p}}^{2}=0.67\right)$. It was significantly higher for error feedback compared to correct feedback. These results are shown in Fig. 2c. 2.3.2.2. P300 We can observe on head maps that the surface Laplacian permitted the scalp diffusing activity to be removed, and clearly delimited the P300 activity (Fig. 3). In accordance with monopolar data, we observed a main effect of difficulty on the CSD amplitude of the P300 $\left(\mathrm{F}(1,16)=7.91, \mathrm{p}<.05, \eta_{\mathrm{p}}^{2}=0.33\right)$. The CSD amplitude of the P300 was again significantly higher in the difficult condition than in the easy condition $(\mathrm{p}<.01)$. These results are shown in Fig. 2d. For results with a baseline taken during the fixation square, see Supplementary materials.

\subsection{Correlations}

The Spearman rank correlation coefficients obtained between all response-locked and feedback-locked potentials are reported in Table 2. They have been computed for both grand average and CSD amplitude measures. We can observe that there is only a significant correlation between the P300 amplitude and both the Pe/Pc and the FRN. After the surface Laplacian transformation, only a tendency towards a negative correlation $(\rho=-0.23, \mathrm{p}=.06)$ was observed between the $\mathrm{Pe} / \mathrm{Pc}$ and the P300 components.

Table 2

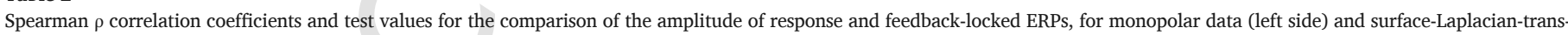
formed data (right side). Bold values indicate significant correlations.

\begin{tabular}{|c|c|c|c|c|c|c|c|}
\hline & \multicolumn{3}{|c|}{ Monopolar measures } & \multicolumn{4}{|c|}{ Surface Laplacian transformation } \\
\hline & ERN/CRN & $\mathrm{Pe} / \mathrm{Pc}$ & FRN & ERN/CRN & $\mathrm{Pe} / \mathrm{Pc}$ & FRN & \\
\hline ERN/CRN & - & - & - & - & - & - & ERN/CRN \\
\hline $\mathrm{Pe} / \mathrm{Pc}$ & $\begin{array}{l}-0.15 \\
\mathrm{p}=.20\end{array}$ & - & - & $\begin{array}{l}-0.03 \\
p=.78\end{array}$ & - & - & $\mathrm{Pe} / \mathrm{Pc}$ \\
\hline FRN & $\begin{array}{l}-0.15 \\
p=.24\end{array}$ & $\begin{array}{l}0.06 \\
p=.63\end{array}$ & - & $\begin{array}{l}-0.17 \\
p=.18\end{array}$ & $\begin{array}{l}0.20 \\
\mathrm{p}=.11\end{array}$ & - & FRN \\
\hline P300 & $\begin{array}{l}0.19 \\
p=.12\end{array}$ & $\begin{array}{l}-0.33 \\
p<.01\end{array}$ & $\begin{array}{l}-0.25 \\
p<.05\end{array}$ & $\begin{array}{l}0.15 \\
\mathrm{p}=.22\end{array}$ & $\begin{array}{l}-0.23 \\
p=.06\end{array}$ & $\begin{array}{l}-0.10 \\
p=.42\end{array}$ & P300 \\
\hline
\end{tabular}




\section{Discussion}

The aim of this ERP study was to assess the effect of task difficulty on performance monitoring event-related potentials time-locked to response onset (ERN/CRN and Pe/Pc) and to feedback onset (FRN and P300), and to then discuss the functional significance of these different ERPs. We used a vertically-oriented arrowhead version of the Eriksen flanker task with feedback. The level of task difficulty was manipulated by using the flankers in the difficult condition, whereas they were not present in the easy condition. This was associated with a fast stimulus display and high time-pressure in both conditions.

The key results of this study are the following: i) We replicated, with a modified task, results of both behavioral measures and the impact of difficulty on response-locked performance monitoring ERPs (ERN/CRN $-\mathrm{Pe} / \mathrm{Pc}$ ); ii) We observed a differential impact of accuracy and difficulty on fronto-central and centro-parietal performance monitoring ERPs; iii) We observed complementing modulations between centro-parietal components (i.e., $\mathrm{Pe} / \mathrm{Pc}$ and $\mathrm{P} 300$ ), but not between fronto-central components (i.e., ERN/CRN and FRN) ERPs, and iv) the use of the surface Laplacian helped us to better dissociate the various ERPs associated with performance monitoring and to refine the analysis of their sensitivity and role. We will discuss these different results in turn in the following sections.

\subsection{Difficulty and behavioral measures}

The various behavioral results obtained are in accordance with the performance monitoring literature and the results usually reported for the flanker task (Braem et al., 2015; Van der Borght et al., 2016). We observed that participants responded significantly faster when they committed errors than when they made correct responses. They also responded significantly faster for congruent compared to incongruent trials, in the difficult condition. Moreover, an effect of difficulty has been well observed. Indeed, we showed longer reaction times in the difficult condition (congruent and incongruent trials) than in the easy condition for both error and correct trials. We also observed higher error rates in the difficult incongruent condition than in the difficult congruent and easy conditions. Interestingly, these results, even though quite anticipated, were not obvious. Indeed, most of the literature using modified versions of the flanker task has focused on displaying of the stimuli and flankers horizontally. We displayed these vertically in our task as a first step in order to further adapt the task to a more applied, operational context of study, such as aeronautics-related environments, e.g., during the take-off or landing of a plane, or during air-traffic control situations. In these situations, data are provided, among others, to increase or lower height (go up or down).

\subsection{Difficulty and response-locked ERPs with and without a surface Laplacian transformation}

Concerning the effect of difficulty on ERPs, we partially reproduced results reported by Van der Borght et al. (2016). We have shown that there is an effect of task accuracy, modulated by difficulty, on response-locked potentials (ERN/CRN and Pe/Pc). We observed a significant effect of difficulty on the monopolar Pe/Pc amplitude and an accuracy $\mathrm{x}$ difficulty interaction effect on the monopolar ERN/CRN and $\mathrm{Pe} / \mathrm{Pc}$ amplitudes. The Pe/Pc amplitude was higher in the easy condition than in the difficult condition. The difference wave between errors and correct responses for the negative (ERN-CRN) and positive (Pe-Pc) components was also higher in the easy condition than in the difficult condition. At first sight, both results can be explained in terms of error salience. Indeed, several studies have shown an impact of error salience on the ERN component, but not on the CRN, and on the Pe/Pc components (Hajcak et al., 2005; Riesel et al., 2012). In our case, we could argue that error salience increases in the easy condition, and is lower in the difficult condition. The salience can be reflected by the increased difference wave amplitude that we observed at $\mathrm{FCz}$ and $\mathrm{CPz}$ in the easy condition compared to the difficult one. In addition, our results suggest a higher difficulty effect on $\mathrm{Pe} / \mathrm{Pc}$ components. The decrease in both difference waves (ERN-CRN and Pe-Pc) can also be explained with regard to uncertainty. Pailing and Segalowitz (2004) and Scheffers and Coles (2000) suggest that an increase in uncertainty, modified by perceptual difficulty as opposed to response mapping, attenuates the ERN and increases the CRN. Likewise, Endrass et al. (2012) demonstrated an attenuation of the centro-parietal (Pe/Pc) component with increasing perceptual difficulty.

However, different results were observed when using the surface Laplacian. First, the main difficulty effect on monopolar Pe/Pc amplitude disappeared. Then, the accuracy $\mathrm{x}$ difficulty interaction effect observed on the monopolar ERN/CRN amplitude also vanished after the surface Laplacian transform. The surface Laplacian allows the EEG data to be spatially filtered and thus improves the definition and localization of ERP components, by reducing the current diffusion induced by the skull (increasing both the spatial and temporal resolution) (Burle et al., 2015; Carvalhaes and de Barros, 2015). Applied to performance monitoring data from $\mathrm{FCz}(\mathrm{ERN} / \mathrm{CRN})$ and $\mathrm{CPz}(\mathrm{Pe} / \mathrm{Pc})$ electrodes, this transformation removes a positive wave, namely the early $\mathrm{Pe} / \mathrm{Pc}$ component (Van der Borght et al., 2016). In our study, the removal of this early $\mathrm{Pe} / \mathrm{Pc}$ component, which is affected by difficulty according to Van der Borght et al. (2016), may justify the loss of the difficulty effect observed on both the ERN/CRN and Pe/Pc components with the monopolar data. In contrast, the simple difficulty effect observed on the monopolar $\mathrm{Pe}$ amplitude for erroneous responses in the accuracy $\mathrm{x}$ difficulty interaction remained significant after the surface Laplacian was applied. The significant effect of difficulty on the difference between Pe and Pc was also maintained. This result seems to be opposite to that of most studies on the effect of error salience or uncertainty on response-locked potentials. However, this result difference may be justified by the fact that, in most studies on error awareness, researchers only measured monopolar grand averages, thus assessing the effect of error salience on the ERN/ $\mathrm{CRN}$ and the early Pe/Pc component at the same time. Finally, part of our results are not consistent with those obtained by Van der Borght et al. (2016) who used a surface Laplacian transform. Their study, as they mention in their discussion, manipulated both motor response mapping difficulty and perceptual difficulty. In our case, only perceptual difficulty was manipulated through the addition of flankers in the difficult condition, and time pressure in both conditions. Response mapping remained unchanged. This can justify some differences observed in our results.

One may argue that instead of clarifying the activity of the various performance-monitoring components, the surface Laplacian transformation only removes parts of this activity. However, the surface Laplacian transformation has proven to be very helpful in dissociating and separately analyzing performance-monitoring components, both at fronto-central locations where ERPs are quite localized, and at centro-parietal locations where broader ERPs are more diffuse (Twomey et al., 2015; Vidal et al., 2003). It also has the up side of providing reference-free data. Other analysis techniques can be used to isolate the components, such as the PCA or ICA. However, as previously reported and argued by several authors, most of these analyses are purely statistical analyses and do not rely on any physiological meaning (Kayser and Tenke, 2015a; Van der Borght et al., 2016). These techniques also very often require biophysical assumptions concerning tissue conductivity and geometry (Kayser and Tenke, 2015b, 2015a), and can lead to non-physiological outcomes (Delorme et al., 2012).

Taken together, data suggest that i) the ERN and CRN components are not modulated by task difficulty, contrary to the Pe component; ii) 
the difficulty effect on the Pe component can be explained by the error awareness functional theory and the more salient errors in the easy condition, but does not seem to apply to the ERN/CRN component; and iii) the use of the surface Laplacian for response-locked performance monitoring ERP analysis appears to be relevant for improving our understanding about their functional significance.

\subsection{Difficulty and feedback-locked ERPs with or without a surface Laplacian transformation}

A second major concern was the impact of the difficulty on feedback-locked potentials. Our results revealed that the FRN-P300 complex seems to reproduce the same pattern as the ERN-Pe/Pc. Indeed, we observe, both before and after the application of the surface Laplacian, that the FRN component is modulated by accuracy and not by difficulty. This result is consistent with several studies on feedback-related components, which showed that this ERP responded to a worse-than-expected feedback stimulus (Donkers and Van Boxtel, 2005; Holroyd et al., 2006; Oliveira et al., 2007). No effect of the task difficulty has ever been shown on the FRN, to our knowledge. This result is consistent with both the Reinforcement Learning and Reward Positivity theories. Indeed, feedback stimuli are equivalent in both difficulty conditions and there is no monetary reward associated with them. Thus, what we observe is either a negative activity linked to a worse than expected response (negative feedback), or a positive activity linked to a good response. On the other hand, the centro-parietal potential P300 seems to be sensitive to the difficulty level of the stimulus. This result is also consistent with the most accepted theory regarding this ERP: it is now well established that the P300 amplitude is related to the quantity of information extracted from the stimulus (Johnson and Donchin, 1978; Sato et al., 2005). This functional significance is supported by our results, which show a lower amplitude of the P300 in the easy condition compared to the difficult one, with and without a surface Laplacian transformation. Indeed, in our study, information provided to the subject by feedback was much more important in the difficult condition due to the smaller salience of the congruent or incongruent stimulus. Feedback information was more useful or relevant to assess the accuracy of the trial. Finally, our results show that the surface Laplacian does not seem to have a major impact on feedback-locked potentials. Although we observe a more accentuated pattern of the ERPs for the FCz electrode, the variations of the potentials are constant.

\subsection{Relationship between performance monitoring components}

The relationship between the four performance monitoring components is not completely understood at the moment. Our results help to clarify the debate regarding the link between the ERN and the FRN, as well as regarding the role of the Pe/Pc and P300 components. They show that after the surface Laplacian transformation, both the ERN/ CRN and FRN are only modulated significantly by the accuracy of the response, or of the feedback stimulus respectively. Conversely, both the $\mathrm{Pe} / \mathrm{Pc}$ and P300 are significantly modulated by the difficulty level of the task: by an interaction between accuracy and task difficulty for the $\mathrm{Pe} / \mathrm{Pc}$, and as a main effect for P300. However, the correlation analyses did not allow us to formalize a precise link. Indeed, after the surface Laplacian transformation, no significant correlation was observed between any of the components. Only the amplitude of the Pe/Pc tend to correlate with the P300 amplitude. The lack of correlation (or low correlation) suggests that there is no direct dependency between the variations of the components at a quantitative level, but it does not exclude the fact that the components can be jointly modulated by a same factor, i.e., they can contribute to a common functional role. A different sensitivity to other factors of these different components (i.e., another more specific functional role) may help to justify the lack of cor- relation. On the one hand, authors have argued that there is some kind of duality in the ERN and FRN components (Gentsch et al., 2009; Holroyd and Coles, 2002). Particularly, several authors have proposed the hypothesis that the ERN and FRN amplitude are complementary and could vary in an inversely proportional manner. Gentsch et al. (2009) for example, hypothesized that the "[...] FRN occurs only when internal error detection based on mismatch or conflict of correct and incorrect response tendencies does not provide sufficient certainty about the action outcome". They also showed an inverse relationship between the amplitudes of both ERPs. Based on this literature, we should observe a variation of the ERN and FRN amplitudes across difficulty conditions. However, we have shown with this study that both the ERN and FRN are not affected by task difficulty. Nevertheless, we have to take into account that their protocol varied from ours, since visual feedback was only provided for correct responses, but not for errors. Moreover, we have to point out the fact that the use of averaging in our study, could mask those variations. An interesting line of study could be to assess the amplitude variations between the ERN/CRN and FRN at the trial level.

On the other hand, it is argued that the $\mathrm{Pe} / \mathrm{Pc}$ component could correspond either to a P300 component specific to performance monitoring (Falkenstein et al., 1991; O'Connell et al., 2007; Ridderinkhof et al., 2009), or that it could be a manifestation of error awareness (Nieuwenhuis et al., 2001; Rigoni et al., 2015; Scheffers and Coles, 2000; Vidal et al., 2000). Both theories account for the fact that the P300 component is not modulated by accuracy, contrary to the Pe/ Pc component. Considering the hypothesis that the Pe/Pc ERP corresponded to a P300 component, a main effect of difficulty would be expected on the Pe/Pc amplitude after the surface Laplacian transformation, or at least a significant amplitude difference in the Pc component between the easy condition and the difficult one. Indeed, the quantity of information extracted from the stimulus is higher, in terms of errors, in the difficult condition compared to the easy one. However, this is not the case. This role, even though not assessed in the current study, seems to correspond more to the early $\mathrm{Pe} / \mathrm{Pc}$ component, which is removed by the surface Laplacian (Van der Borght et al., 2016). Thus, our results seems to not support this first hypothesis, but rather the second, error awareness hypothesis. The Pe/Pc has a higher CSD amplitude in the easy condition than in the difficult one, and for errors compared to correct responses. In our task, the increase in difficulty triggers a decrease in error salience. This decrease can be related to a lower error awareness and is thus consistent with the error awareness theory (Nieuwenhuis et al., 2001). Moreover, another study showed that Pe/Pc's amplitude was influenced by the error rate difference between difficulty conditions (Endrass et al., 2012). Given that our difficult condition induced at least four times more errors than the easy condition, it is also not surprising that the amplitude of the Pe/Pc component is modulated by the difficulty.

Finally, our results show that the complementarity between response-locked and feedback-locked ERPs is more attributable to the Pe/ Pc and the P300 components. Given the role of these two potentials, we assume that the $\mathrm{P} 300$, as following the $\mathrm{Pe} / \mathrm{Pc}$ in a response-feedback protocol, can be used as a reinforcement signal. In an easy condition with high error salience, error awareness is higher; there is no need to recover information from the feedback stimulus. Conversely, in a difficult condition with low error salience, feedback is of major relevance to uncover the exact nature of the trial; this condition thus requires more information to be extracted from the feedback stimulus. Given this theory, it is fair to observe that the Pe is higher in the easy condition than in the difficult condition and vice-versa for the following P300, which seems to be the case in our study. In order to reach a clear conclusion on the relationships between the ERN/CRN and FRN, and on the duality between the Pe/Pc and P300, it would be interesting to assess the variations at the trial level. 


\section{Conclusion}

In this paper we have shown that performance monitoring potentials ERN/CRN, Pe/Pc, FRN and P300 respond differently to difficulty level modulations in a modified, vertically-oriented, version of the flanker task with feedback. The use of a surface Laplacian signal-processing technique has allowed us to improve our understanding of the role of various ERP components associated with performance monitoring. We found that they can be classified into two categories according to their topography: the fronto-central ERPs (ERN/CRN and FRN), which are only affected by accuracy, and centro-parietal potentials ( $\mathrm{Pe} / \mathrm{Pc}$ and P300), which respond to difficulty. Measuring the impact of difficulty levels on feedback-locked components had never been done, to our knowledge. This study allowed us to show that the duality and complementarity between response-locked and feedback-locked performance monitoring ERPs could not be observed on fronto-central components, but seemed to be observed on centro-parietal components. Further study is required to assess how this relationship can be defined and analyzed.

\section{Methods and materials}

This research was approved by a local French ethics committee (Comité d'Ethique pour les recherches non interventionnelles de Grenoble CERNI - IRB number: IRB00010290-2016-09-13-12) and conducted according to the principles expressed in the Declaration of Helsinki.

\subsection{Participants}

Based on the performance monitoring literature (including between 15 and 20 participants) (Holroyd and Coles, 2002; Oliveira et al., 2007) and on a power analysis of literature data (suggesting a sample size of 4 and 8 participants to detect the ERN and the Pe, respectively) (Van der Borght et al., 2016), seventeen healthy right-handed participants (12 men; 27.5 years \pm 4.78 years) were recruited from the general population to perform the experiment. They were all naïve to the task. Their laterality was measured with the Edinburgh inventory test (Oldfield, 1971). They had normal or corrected-to-normal vision and hearing, had no neurological or psychiatric disorders and were not under any medication (as reported through a questionnaire before the experiment). They signed a written informed consent and received a financial compensation.

\subsection{Experimental task and procedure}

\subsubsection{Stimuli}

Task stimuli were displayed in white against a black background, using the E-prime 2.0 software (E-prime Psychology Software Tools Inc., Pittsburg, USA) on a 19-in CRT monitor (with a $1024 \times 768$ pixel resolution and a $100-\mathrm{Hz}$ refresh rate) located $46 \mathrm{~cm}$ away from the participant in an unlit room. They consisted of five vertically-oriented arrowheads $\left(2.8^{\circ} \times 0.6^{\circ}\right.$ of visual angle) that included a target (central arrowhead) and four flankers ( 2 arrowheads above and below the target). Two difficulty levels were considered. The easy condition only displayed the target arrowhead $\left(0.5^{\circ} \times 0.6^{\circ}\right.$ of visual angle). The target arrowhead could either be pointing up, or down. The difficult condition displayed the target flanked above and below. The flanker arrowheads all pointed in the same direction, but could either be congruent with the target arrowhead (in the same direction) or incongruent (in the opposite direction). Fig. 3a illustrates the different stimulus configurations.

Three feedback stimuli (see Fig. 3b) were used. If the participant responded within a given time interval $(550 \mathrm{~ms})$, feedback displayed the expected response surrounded by a rectangle $\left(8.26^{\circ} \times 10.9^{\circ}\right.$ of visual angle), which could be either green for a participant's correct response or red for a participant's erroneous response. If the participant did not respond within the given time interval, the feedback stimulus was the word "!!!RETARD!!!" (for “!!!LATE!!!” in French).

\subsubsection{Protocol}

Participants took part in a modified flanker task (Eriksen and Eriksen, 1974) with feedback. They had to identify, as quickly and as accurately as possible, the orientation of the target (i.e., up or down) using a response box (Chronos ${ }^{\circledR}$ Psychology Software Tools Inc., Pittsburg, USA), on which response buttons were one above the other representing up and down orientations. The experiment was divided into two difficulty sessions (easy and difficult), separated by a break. The session order was counterbalanced between participants. Each session was composed of ten blocks separated by breaks. In the easy condition, each block included 72 trials (lasting $3.5 \mathrm{~min}$ ): 36 with the target heading up and 36 with the target heading down, pseudo-randomly presented. In the difficult condition, each block included 48 trials (lasting $2.2 \mathrm{~min}$ ), and 4 types of stimuli (congruent up and down, incongruent up and down) were equiprobable and pseudo-randomly presented. Thus, the whole experiment displayed a total of 720 trials in the easy condition and 480 trials in the difficult condition (a total of 1200 trials per participant) and lasted approximately one hour.

For both conditions, each trial started with the display of a fixation rectangle $\left(4.85^{\circ} \times 1.9^{\circ}\right.$ of visual angle) for a variable duration ( $1 \pm 0.25 \mathrm{~s}$ ), followed by the display of the stimulus for $10 \mathrm{~ms}$, then a fixation point for $540 \mathrm{~ms}$. The participant had at the most $550 \mathrm{~ms}$ $\left(\mathrm{t}_{\text {stimulus }}+\mathrm{t}_{\text {fixation point }}\right)$ to state whether the target was pointing up or down, by pressing the corresponding response key with the right thumb or index. This time pressure allowed error promoting (Falkenstein et al., 2000, 1991; Hohnsbein et al., 1991). Then, if the participant responded on time (i.e., within the $550 \mathrm{~ms}$ interval), his/her response was displayed as one arrowhead going in the chosen direction $(350 \mathrm{~ms})$, followed by a black screen for a variable duration (randomized between 300 and $350 \mathrm{~ms}$ ). If the participant did not respond on time, the fixation point stayed on the screen until the jitter black screen was displayed. Finally, feedback was given to the participant for $500 \mathrm{~ms}$. Participants were previously familiarized with the task for each experimental condition. No training trial was analyzed. Fig. 4 shows a complete description of a trial.

The stimulus display duration and time pressure at response time were both chosen in order to promote errors and induce a sufficient number of error trials for the EEG data analysis. These methodological choices, as well as the number of trials in each difficulty condition, were identified on the basis of pretests that we carried out, so as to reach a minimum of $15 \%$ of errors in the difficult condition for the used task, and an equivalent number of error trials in the easy condition.

\subsection{Measure and analysis}

\subsubsection{Behavioral}

Reaction times (RT) and response accuracy (Error Rates - ER) for target identification were recorded for each trial using the E-Prime 2.0 software (Psychology Software Tools, Inc., Pittsburg, USA) and analyzed using the R software (R Development Core Team, 2008). Trials with RT faster than $100 \mathrm{~ms}$ were cosnsidered as motor anticipation and were removed from data. Given the task instructions (i.e., to respond within $550 \mathrm{~ms}$ post stimulus onset), trials with RT slower than $550 \mathrm{~ms}$ were considered as late responses; they were thus also removed (3.6\% of total number of trials). The mean RT of the remaining trials (i.e., 11,737 trials in the easy condition and 7665 trials in the difficult condition) were analyzed using a two-way repeated measures ANOVA with 


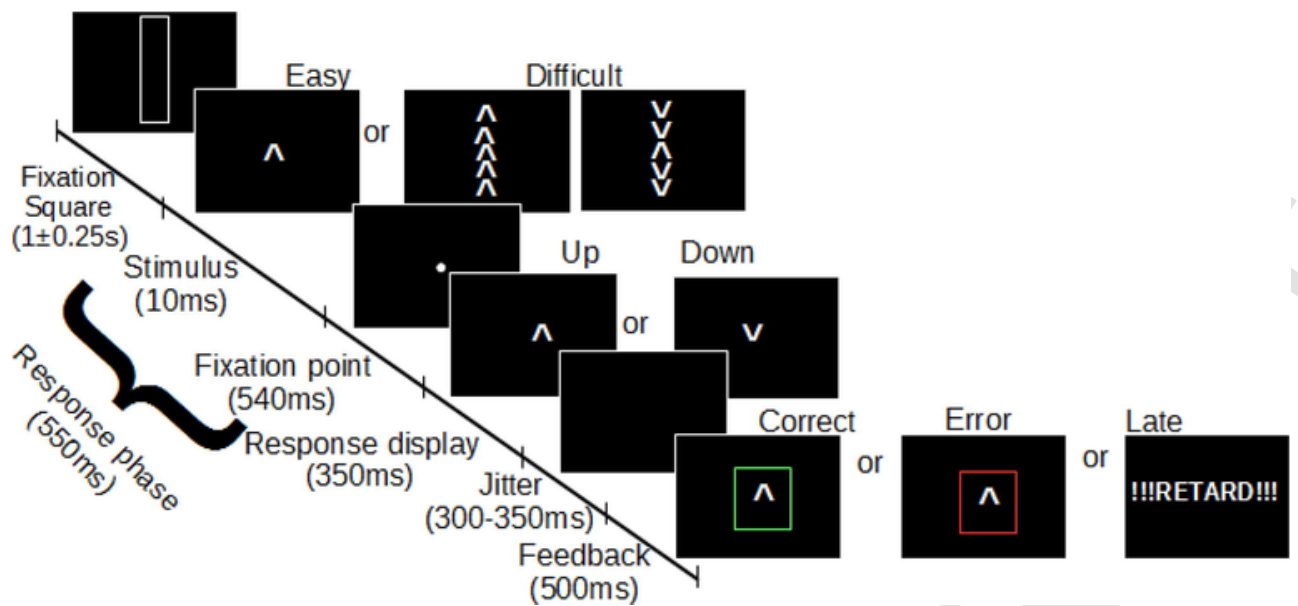

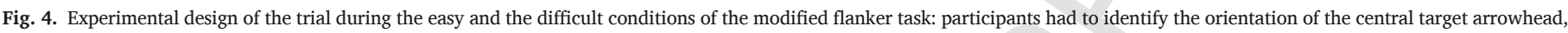

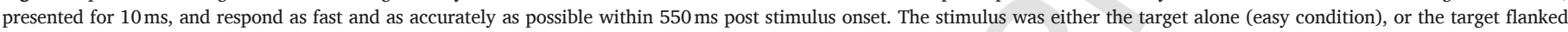
above and below by arrowheads going in the same (congruent) or the opposite (incongruent) direction. They were given feedback on their performance at the end of every trial.

difficulty (easy vs. difficult congruent vs. difficult incongruent) and accuracy (error vs. correct response) as within-subject factors. A Greenhouse-Geisser correction was applied when necessary, and all results were reported after that correction if performed (as well as the Greenhouse-Geisser epsilon value). Partial eta squared was provided as a measure of the effect size. Mean comparisons were performed using a Tukey HSD test.

Response accuracy was measured for each condition as the ratio between the number of error trials and the total number of trials in the condition. The mean accuracy was analyzed using a repeated measures ANOVA with difficulty (easy vs. difficult congruent vs. difficult incongruent) as within-subject factor. A Greenhouse-Geisser correction was applied, and all results were reported after that correction if performed (as well as the Greenhouse-Geisser epsilon value). Partial eta squared was provided as a measure of the effect size, since it allows for a better comparison of within-subject designs (Levine and Hullett, 2002; Richardson, 2011). Mean comparisons were performed using a Tukey HSD test. All results were reported as mean \pm SEM. The significance level was set at 0.05 .

\subsubsection{Electroencephalography}

The electroencephalogram (EEG) was continuously recorded using an ActiCAP (Brain Products $\mathrm{GmbH}$ ) equipped with $75 \mathrm{Ag} / \mathrm{AgCl}$ unipolar active electrodes (i.e., the 65 ActiCAP montage to which we added F9, F10, P9, P10, PO9, PO10, O9, O10, M1, M2), which were positioned according to the extended 10-20 system (Jasper, 1958). The reference and ground electrodes used for the EEG data acquisition were positioned on the forehead (respectively AFz and Fpz electrodes). Blinks and eye movements were also monitored using four pure silver electro-oculography (EOG) electrodes: two positioned above and below the left eye on the median axis for vertical activities and two at the outer canthi of the eyes for horizontal activities. The ground electrode for the EOG data was placed on the right earlobe. In addition, participants were instructed to limit blinking and eye-movements. The signal's impedance was kept below $10 \mathrm{k} \Omega$ for all electrodes. The signal was amplified using an ActiCHamp ${ }^{\mathrm{TM}}$ system (Brain Products, Inc.), digitized at a 24-bit rate and sampled at $1000 \mathrm{~Hz}$, with a $0.05 \mu \mathrm{V}$ resolution.

All EEG data analyses were performed using EEGLAB (Delorme \& Makeig, 2004) and Fieldtrip (Oostenveld, Fries, Maris, \& Schoffelen, 2011) MATLAB $\AA$ toolboxes (The MathWorks, Inc.). The raw EEG data were re-referenced offline to the linked mastoids. The signal was segmented into epochs from trial onset (around $3000 \mathrm{~ms}$ ); each epoch included the response and feedback periods. The signal was then down- sampled at $500 \mathrm{~Hz}$ and band-pass filtered between 0.5 and $30 \mathrm{~Hz}$. All segments contaminated with muscular activity and/or non-physiological artifacts were rejected offline after a visual inspection. Artifacts related to ocular movements (saccades and blinks) were corrected by using an Independent Component Analysis (ICA). Two measures of event-related potentials (ERP) were then considered: the ERPs time-locked to the participant's response and to feedback presentation. The "Late" trials were excluded from the data. For all ERP analyses, difficult trials were not separated considering the congruency of the stimulus for robustness purposes of the EEG analysis.

For response-locked potentials, EEG segments were re-segmented into epochs, from $500 \mathrm{~ms}$ before the response to $600 \mathrm{~ms}$ after the response. Data were visually inspected again to remove any remaining periods with artifacts and were then baseline-corrected from 500 to $0 \mathrm{~ms}$ preceding the response (Koban et al., 2010) ${ }^{2}$. Our analysis then focused on both the error-related negativity or the correct-related negativity (ERN/CRN) and the error positivity or correct positivity (Pe/Pc), according to accuracy, which were identified based on the grand averaged data. The ERN and CRN were respectively observed for error and correct trials, between 0 and $100 \mathrm{~ms}$ from the response onset, and measured at the amplitude peak of the component, i.e., the FCz electrode, in accordance with the literature (Gehring et al., 2011). For each participant, the mean amplitudes (between 0 and $100 \mathrm{~ms}$ ) of the ERN (error trials) and CRN (correct trials) were calculated at the FCz electrode for each difficulty level - easy and difficult. The Pe and Pc were respectively observed for error and correct trials, between 200 and $400 \mathrm{~ms}$ from response onset, and measured at the $\mathrm{CPz}$ electrode in accordance with the literature (Grützmann et al., 2014; O'Connell et al., 2007). For each participant, mean amplitudes (between 200 and $400 \mathrm{~ms}$ ) of the Pe (error trials) and Pc (correct trials) were calculated at the $\mathrm{CPz}$ electrode for each difficulty level - easy and difficult.

For feedback-locked potentials, EEG segments were re-segmented into epochs from $200 \mathrm{~ms}$ before feedback onset to $750 \mathrm{~ms}$ after feedback onset. Data were visually inspected again to remove any remaining periods with artifacts. Baseline correction was applied, from 200 to $0 \mathrm{~ms}$ preceding feedback. Our analysis then focused on both the feed-

\footnotetext{
2 This baseline may seem unusual at first sight, but was selected on purpose to lower the putative impact of pre-motor components on response-locked ERPs. Moreover, to ensure the validity of the baseline, the same data were baseline-corrected using a baseline taken in the time-window of the fixation square (from $900 \mathrm{~ms}$ to $600 \mathrm{~ms}$ before response and from $1650 \mathrm{~ms}$ to $1350 \mathrm{~ms}$ before feedback); we observed very similar results, as shown in supplementary materials.
} 
back-related negativity (FRN) and the P300 component, which were identified based on the grand averaged data. The FRN was measured using a base-to-peak method developed by Oliveira et al. (2007) based on an ERP complex observed between 160 and 500 ms from feedback onset at the FCz electrode, in accordance with the literature (Donkers and Van Boxtel, 2005; Sambrook and Goslin, 2015; Yu and Zhou, 2006). The ERP complex includes a positive component (Pos1) between 160 and $270 \mathrm{~ms}$, followed by a negative component (Neg1) up to $350 \mathrm{~ms}$ and then a positive component (Pos2) up to $500 \mathrm{~ms}$. The base-to-peak method consists in identifying the amplitude peak value of each component, and calculating the voltage difference between the value obtained for the negative component ( Neg 1 ) and the average of the values obtained for the positive components (Pos1 and Pos2). The result obtained corresponds to the FRN amplitude that was calculated for each difficulty level - easy and difficult - and feedback accuracy (correct and error; the "LATE" responses are not considered here) and for each participant. The amplitude peak values were defined using the ERPLAB toolbox (Lopez-Calderon and Luck, 2014) and the local peak characterization function (with the "Neighborhood" parameter equal to 2). The P300 was observed between 200 and 400 ms post-feedback onset and measured at the $\mathrm{CPz}$ electrode, in accordance with the literature $\mathrm{Wu}$ and Zhou, 2009). For each participant, the mean amplitude (between 200 and $400 \mathrm{~ms}$ ) of the P300 was calculated for each difficulty level easy and difficult - and feedback accuracy (correct and error).

All ERP components' amplitudes were measured as monopolar ERP recordings and after the surface Laplacian method was applied. For each participant, the surface Laplacian was performed using a spherical spline algorithm (Perrin et al., 1989), as implemented in the CSD toolbox of MATLAB (Tenke and Kayser, 2012). The surface Laplacian corresponds to the second spatial derivative of a potential at a certain point (Carvalhaes and de Barros, 2015). Carvalhaes and de Barros (2015) show that it assesses the presence of a current source, or electrical activities on the dura surface. As reported previously, this technique reduces the current diffusion induced by the skull (i.e., reducing spatial noise and increasing spatial resolution) (Carvalhaes and de Barros, 2015; Tenke and Kayser, 2012; Van der Borght et al., 2016). It also requires no assumption, except from local isotropicity of the scalp. Finally, an approximation of the corticogram can be provided by this technique due to its relation to electrical activities on the dura surface (Gevins, 1989; Nunez and Srinivasan, 2006). In our analysis, we used the default parameters, i.e., smoothing constant $=1.10^{-5}$, head radius $=10 \mathrm{~cm}$, spline $=5$. This method has been used previously on several performance monitoring ERPs and permitted to uncover the existence of the Correct-Related Negativity (CRN) and correct Positivity (Pc), as well as to isolate the early from the late error Positivity (Pe) among other results (Allain et al., 2004; Van der Borght et al., 2016; Vidal et al., 2003).

Mean ERP amplitudes, monopolar or surface-Laplacian-transformed, were statistically analyzed using a two-way repeated measures ANOVA, with accuracy/feedback (error vs. correct) and difficulty (easy vs. difficult) as within-subject factors. Partial eta squared was provided as a measure of the effect size. Mean comparisons were explored using the Bonferroni post-hoc test (for multiple comparisons) and a significance threshold set at 0.05 .

Correlation tests were performed with Spearman's $\rho$ coefficient on the mean amplitudes of all components, for all participants. Tests were performed with the R software (R Development Core Team, 2008) on monopolar recordings and CSD amplitudes. $\rho$ coefficients and p-values are reported in Table 2, and we chose a significance threshold of 0.05 .

\section{Acknowledgements}

We would like to thank AWS traduction for their proofreading service.

\section{Funding}

This work was supported by a Région Provence-Alpes-Côte d'Azur grant (Emploi Jeunes Doctorants) and a grant from the Agence Nationale de la Recherche (Young Researcher Program ANR-15-CE26-0010-01)

\section{Conflict of interest}

None.

\section{Contributors}

$\mathrm{BS}, \mathrm{AC}$ and $\mathrm{BB}$ designed the experimental procedure. $\mathrm{BS}, \mathrm{AC}$ and $\mathrm{AD}$ analyzed the data. $\mathrm{BS}$ wrote the manuscript. $\mathrm{AC}, \mathrm{AD}$ and $\mathrm{BB}$ read and critically reviewed the manuscript.

All authors are in agreement with the latest version of the manuscript.

\section{Reprints}

Correspondence and requests for reprints should be addressed either to the corresponding author (Bertille Somon), or to Bruno Berberian, ONERA, The French Aerospace Lab, 13661 Salon Cedex Air.

\section{Appendix A. Supplementary data}

Supplementary data to this article can be found online at https:// doi.org/10.1016/j.brainres.2018.10.007.

\section{References}

Alexander, W.H., Brown, J.W., 2010. Computational models of performance monitoring and cognitive control. Top. Cogn. Sci. 2, 658-677. https://doi.org/10.1111/j. 1756-8765.2010.01085.x.

Alexander, W.H., Brown, J.W., 2011. Medial prefrontal cortex as an action-outcome predictor. Nat. Neurosci. 14, 1338-1344. https://doi.org/10.1038/nn.2921.

Allain, S., Carbonnell, L., Falkenstein, M., Burle, B., Vidal, F., 2004. The modulation of the Ne-like wave on correct responses foreshadows errors. Neurosci. Lett. 372, 161-166. https://doi.org/10.1016/j.neulet.2004.09.036.

Braem, S., Coenen, E., Bombeke, K., van Bochove, M.E., Notebaert, W., 2015. Open your eyes for prediction errors. Cogn. Affect. Behav. Neurosci. 15, 374-380. https://doi. org/10.3758/s13415-014-0333-4.

Burle, B., Roger, C., Allain, S., Vidal, F., Hasbroucq, T., 2008. Error negativity does not reflect conflict: a reappraisal of conflict monitoring and anterior cingulate cortex activity. J. Cogn. Neurosci. 20, 1637-1655. https://doi.org/10.1162/jocn.2008.20110.

Burle, B., Spieser, L., Roger, C., Casini, L., Hasbroucq, T., Vidal, F., 2015. Spatial and temporal resolutions of EEG: is it really black and white? A scalp current density view. Int. J. Psychophysiol. 97, 210-220. https://doi.org/10.1016/j.ijpsycho.2015.05.004.

Carvalhaes, C., de Barros, J.A., 2015. The surface Laplacian technique in EEG: theory and methods. Int. J. Psychophysiol. 97, 174-188. https://doi.org/10.1016/j.ijpsycho. 2015.04.023.

Cavanagh, J.F., Frank, M.J., 2014. Frontal theta as a mechanism for cognitive control Trends Cogn. Sci. 18, 414-421. https://doi.org/10.1016/j.tics.2014.04.012.

Dehaene, S., Posner, M.I., Tucker, D.M., 1994. Localization of a neural system for error detection and compensation. Psychol. Sci. 303-305.

Delorme, A., Palmer, J., Onton, J., Oostenveld, R., Makeig, S., 2012. Independent EEG sources are dipolar. PLoS One 7, e30135https://doi.org/10.1371/journal.pone. 0030135.

Donkers, F.C.L., van Boxtel, G.J.M., 2005. Mediofrontal negativities to averted gains and losses in the slot-machine task: a further investigation. J. Psychophysiol. 19, 256-262. https://doi.org/10.1027/0269-8803.19.4.256

Endrass, T., Klawohn, J., Preuss, J., Kathmann, N., 2012. Temporospatial dissociation of Pe subcomponents for perceived and unperceived errors. Front. Hum. Neurosci. 6, https: //doi.org/10.3389/fnhum.2012.00178. 
Eriksen, B.A., Eriksen, C.W., 1974. Effects of noise letters upon the identification of a target letter in a nonsearch task. Percept. Psychophys. 16, 143-149. https://doi.org/10. 3758/BF03203267.

Falkenstein, M., Hohnsbein, J., Hoormann, J., Blanke, L., 1991. Effects of crossmodal divided attention on late ERP components. II. Error processing in choice reaction tasks. Electroencephalogr. Clin. Neurophysiol. 78, 447-455. https://doi.org/10.1016/ 0013-4694(91)90062-9.

Falkenstein, M., Hoormann, J., Christ, S., Hohnsbein, J., 2000. ERP components on reaction errors and their functional significance: a tutorial. Biol. Psychol. 51, 87-107. https://doi.org/10.1016/S0301-0511(99)00031-9.

Gehring, W.J., Coles, M.G.H., Meyer, D.E., Donchin, E., 1990. The error-related negativity: an event-related brain potential accompanying errors. Psychophysiology 27, S34.

Gehring, W.J., Liu, Y., Orr, J.M., Carp, J., 2011. The Error-Related Negativity (ERN/Ne). https://doi.org/10.1093/oxfordhb/9780195374148.013.0120.

Gehring, W.J., Goss, B., Coles, M.G.H., Meyer, D.E., Donchin, E., 1993. A neural system for error detection and compensation. Psychol. Sci. 4, 385-390. https://doi.org/10.1111/ j.1467-9280.1993.tb00586.x.

Gentsch, A., Ullsperger, P., Ullsperger, M., 2009. Dissociable medial frontal negativities from a common monitoring system for self- and externally caused failure of goal achievement. NeuroImage 47, 2023-2030. https://doi.org/10.1016/j.neuroimage. 2009.05.064.

Gevins, A., 1989. Dynamic functional topography of cognitive tasks. Brain Topogr. 2, 37-56. https://doi.org/10.1007/BF01128842.

Grützmann, R., Endrass, T., Klawohn, J., Kathmann, N., 2014. Response accuracy rating modulates ERN and Pe amplitudes. Biol. Psychol. 96, 1-7. https://doi.org/10.1016/j. biopsycho.2013.10.007.

Hajcak, G., Moser, J.S., Yeung, N., Simons, R.F., 2005. On the ERN and the significance of errors. Psychophysiology 42, 151-160. https://doi.org/10.1111/j.1469-8986.2005. 00270.x.

Hohnsbein, J., Falkenstein, F., Michael, J., Hoormann, J., Blanke, L., 1991. Effects of crossmodal divided attention on late ERP components. I. Simple and choice reaction tasks. Electroencephalogr. Clin. Neurophysiol. 78, 438-446. https://doi.org/10.1016/ 0013-4694(91)90061-8.

Holroyd, C.B., Coles, M.G.H., 2002. The neural basis of human error processing: reinforcement learning, dopamine, and the error-related negativity. Psychol. Rev. 109, 679-709. https://doi.org/10.1037//0033-295X.109.4.679.

Holroyd, C.B., Hajcak, G., Larsen, J.T., 2006. The good, the bad and the neutral: electrophysiological responses to feedback stimuli. Brain Res. 1105, 93-101. https://doi.org/ 10.1016/j.brainres.2005.12.015.

Iannaccone, R., Hauser, T.U., Staempfli, P., Walitza, S., Brandeis, D., Brem, S., 2015. Conflict monitoring and error processing: new insights from simultaneous EEG-fMRI. NeuroImage 105, 395-407. https://doi.org/10.1016/j.neuroimage.2014.10.028.

Johnson, R., Donchin, E., 1978. On how P300 amplitude varies with the utility of the eliciting stimuli. Electroencephalogr. Clin. Neurophysiol. 44, 424-437. https://doi.org/ 10.1016/0013-4694(78)90027-5.

Kayser, J., Tenke, C.E., 2015. Issues and considerations for using the scalp surface Laplacian in EEG/ERP research: a tutorial review. Int. J. Psychophysiol. 97, 189-209. https: //doi.org/10.1016/j.ijpsycho.2015.04.012.

Kayser, J., Tenke, C.E., 2015. On the benefits of using surface Laplacian (current source density) methodology in electrophysiology. Int. J. Psychophysiol. 97, 171-173. https: //doi.org/10.1016/j.ijpsycho.2015.06.001.

Keil, J., Weisz, N., Paul-Jordanov, I., Wienbruch, C., 2010. Localization of the magnetic equivalent of the ERN and induced oscillatory brain activity. NeuroImage 51, 404-411. https://doi.org/10.1016/j.neuroimage.2010.02.003.

Koban, L., Pourtois, G., Vocat, R., Vuilleumier, P., 2010. When your errors make me lose or win: event-related potentials to observed errors of cooperators and competitors. Soc. Neurosci. 5, 360-374. https://doi.org/10.1080/17470911003651547.

Kujawa, A., Smith, E., Luhmann, C., Hajcak, G., 2013. The feedback negativity reflects favorable compared to nonfavorable outcomes based on global, not local, alternatives: feedback negativity reflects global outcomes. Psychophysiology 50, 134-138. https:// doi.org/10.1111/psyp.12002.

Levine, T.R., Hullett, C.R., 2002. Eta squared, partial eta squared, and misreporting of effect size in communication research. Hum. Commun. Res. 28, 612-625. https://doi. org/10.1111/j.1468-2958.2002.tb00828.x.

Lopez-Calderon, J., Luck, S.J., 2014. ERPLAB: an open-source toolbox for the analysis of event-related potentials. Front. Hum. Neurosci. 8, https://doi.org/10.3389/fnhum. 2014.00213.

Luck, S.J., Kappenman, E.S., 2011. The Oxford Handbook of Event-Related Potential Components. Oxford University Press.

Luft, C.D.B., 2014. Learning from feedback: the neural mechanisms of feedback processing facilitating better performance. Behav. Brain Res. 261, 356-368. https://doi.org/10. 1016/j.bbr.2013.12.043.

Luu, P., Tucker, D.M., Derryberry, D., Reed, M., Poulsen, C., 2003. Electrophysiological responses to errors and feedback in the process of action regulation. Psychol. Sci. 14, 47-53. https://doi.org/10.1111/1467-9280.01417.

Miltner, W.H.R., Braun, C.H., Coles, M.G.H., 1997. Event-related brain potentials following incorrect feedback in a time-estimation task: evidence for a "generic" neural system for error detection. J. Cogn. Neurosci. 9, 788-798. https://doi.org/10.1162/jocn. 1997.9.6.788.

Müller, S.V., Möller, J., Rodriguez-Fornells, A., Münte, T.F., 2005. Brain potentials related to self-generated and external information used for performance monitoring. Clin. Neurophysiol. 116, 63-74. https://doi.org/10.1016/j.clinph.2004.07.009.

Nieuwenhuis, S., Ridderinkhof, K.R., Blom, J., Band, G.P., Kok, A., 2001. Error-related brain potentials are differentially related to awareness of response errors: evidence from an antisaccade task. Psychophysiology 38, 752-760. https://doi.org/10.1111/ 1469-8986.3850752.

Nunez, P.L., Srinivasan, R., 2006. Electric Fields of the Brain: The Neurophysics of EEG, second ed. Oxford University Press, Oxford, New York.

O'Connell, R.G., Dockree, P.M., Bellgrove, M.A., Kelly, S.P., Hester, R., Garavan, H., Robertson, I.H., Foxe, J.J., 2007. The role of cingulate cortex in the detection of errors with and without awareness: a high-density electrical mapping study: error awareness. Eur. J. Neurosci. 25, 2571-2579. https://doi.org/10.1111/j.1460-9568.2007.05477.

Oldfield, R.C., 1971. The assessment and analysis of handedness: the Edinburgh inventory. Neuropsychologia 9, 97-113. https://doi.org/10.1016/0028-3932(71)90067-4.

Oliveira, F.T.P., McDonald, J.J., Goodman, D.F.M., 2007. Performance monitoring in the anterior cingulate is not all error related: expectancy deviation and the representation of action-outcome associations. J. Cogn. Neurosci. 19, 1994-2004. https://doi.org/10. 1162/jocn.2007.19.12.1994.

Overbeek, T.J.M., Nieuwenhuis, S., Ridderinkhof, K.R., 2005. Dissociable components of error processing: on the functional significance of the Pe Vis-à-vis the ERN/Ne. J. Psychophysiol. 19, 319-329. https://doi.org/10.1027/0269-8803.19.4.319.

Perrin, F., Pernier, J., Bertrand, O., Echallier, J.F., 1989. Spherical splines for scalp potential and current density mapping. Electroencephalogr. Clin. Neurophysiol. 72, 184-187. https://doi.org/10.1016/0013-4694(89)90180-6.

Proudfit, G.H., 2015. The reward positivity: from basic research on reward to a biomarker for depression: the reward positivity. Psychophysiology 52, 449-459. https://doi.org/ 10.1111/psyp.12370.

Rabbitt, P.M.A., 1966. Errors and error correction in choice-response tasks. J. Exp. Psychol. 71, 264. https://doi.org/10.1037/h0022853.

Rabbitt, P.M.A., 1966. Error correction time without external error signals. Nature 212, 438. https://doi.org/10.1038/212438a0.

Richardson, J.T.E., 2011. Eta squared and partial eta squared as measures of effect size in educational research. Educ. Res. Rev. 6, 135-147. https://doi.org/10.1016/j.edurev. 2010.12.001.

Ridderinkhof, K.R., Ramautar, J.R., Wijnen, J.G., 2009. To $P_{E}$ or not to $P_{E}$ : a P3-like ERP component reflecting the processing of response errors. Psychophysiology 46 531-538. https://doi.org/10.1111/j.1469-8986.2009.00790.x.

Riesel, A., Weinberg, A., Endrass, T., Kathmann, N., Hajcak, G., 2012. Punishment has a lasting impact on error-related brain activity: punishment modulates error monitoring. Psychophysiology 49, 239-247. https://doi.org/10.1111/j.1469-8986.2011. 01298.x.

Rigoni, D., Pourtois, G., Brass, M., 2015. "Why should I care?” Challenging free will attenuates neural reaction to errors. Soc. Cogn. Affect. Neurosci. 10, 262-268. https://doi. org/10.1093/scan/nsu068.

Roger, C., Bénar, C.G., Vidal, F., Hasbroucq, T., Burle, B., 2010. Rostral Cingulate Zone and correct response monitoring: ICA and source localization evidences for the unicity of correct- and error-negativities. NeuroImage 51, 391-403. https://doi.org/10.1016/ j.neuroimage.2010.02.005.

Sambrook, T.D., Goslin, J., 2015. A neural reward prediction error revealed by a meta-analysis of ERPs using great grand averages. Psychol. Bull. 141 (1), 213. https: //doi.org/10.1037/bul0000006

Sato, A., Yasuda, A., Ohira, H., Miyawaki, K., Nishikawa, M., Kumano, H., Kuboki, T., 2005. Effects of value and reward magnitude on feedback negativity and P300. NeuroReport 16, 407-411.

Scheffers, M.K., Coles, M.G.H., 2000. Performance monitoring in a confusing world: error-related brain activity, judgments of response accuracy, and types of errors. J. Exp. Psychol. Hum. Percept. Perform. 26, 141-151. https://doi.org/10.1037/0096-1523. 26.1.141.

Scheffers, M.K., Coles, M.G.H., Bernstein, P., Gehring, W.J., Donchin, E., 1996. Event-related brain potentials and error-related processing: an analysis of incorrect responses to go and no-go stimuli. Psychophysiology 33, 42-53. https://doi.org/10.1111/j 1469-8986.1996.tb02107.x.

Somon, B., Campagne, A., Delorme, A., Berberian, B., 2017. Performance monitoring applied to system supervision. Front. Hum. Neurosci. 11, https://doi.org/10.3389/ fnhum.2017.00360.

Tenke, C.E., Kayser, J., 2012. Generator localization by current source density (CSD): implications of volume conduction and field closure at intracranial and scalp resolutions. Clin. Neurophysiol. 123, 2328-2345. https://doi.org/10.1016/j.clinph.2012.06.005.

Twomey, D.M., Murphy, P.R., Kelly, S.P., O'Connell, R.G., 2015. The classic P300 encodes a build-to-threshold decision variable. Eur. J. Neurosci. 42, 1636-1643. https://doi. org/10.1111/ejn.12936.

Ullsperger, M., Nittono, H., von Cramon, D.Y., 2007. When goals are missed: dealing with self-generated and externally induced failure. NeuroImage 35, 1356-1364. https:// doi.org/10.1016/j.neuroimage.2007.01.026.

Van der Borght, L., Houtman, F., Burle, B., Notebaert, W., 2016. Distinguishing the influence of task difficulty on error-related ERPs using surface Laplacian transformation. Biol. Psychol. 115, 78-85. https://doi.org/10.1016/j.biopsycho.2016.01.013.

Van Veen, V., Carter, C.S., 2002. The anterior cingulate as a conflict monitor: fMRI and ERP studies. Physiol. Behav. 77, 477-482. https://doi.org/10.1016/ S0031-9384(02)00930-7.

Vidal, F., Hasbroucq, T., Grapperon, J., Bonnet, M., 2000. Is the 'error negativity'specific to errors?. Biol. Psychol. 51, 109-128. https://doi.org/10.1016/ S0301-0511(99)00032-0.

Vidal, F., Burle, B., Bonnet, M., Grapperon, J., Hasbroucq, T., 2003. Error negativity on correct trials: a reexamination of available data. Biol. Psychol. 64, 265-282. https:// doi.org/10.1016/S0301-0511(03)00097-8. 
Wu, Y., Zhou, X., 2009. The P300 and reward valence, magnitude, and expectancy in outcome evaluation. Brain Res. 1286, 114-122. https://doi.org/10.1016/j.brainres.2009. 06.032 .

Yeung, N., Holroyd, C.B., Cohen, J.D., 2004. ERP Correlates of feedback and reward processing in the presence and absence of response choice. Cereb. Cortex 15, 535-544. https://doi.org/10.1093/cercor/bhh153.
Yu, R., Zhou, X., 2006. Brain responses to outcomes of one's own and other's performance in a gambling task. NeuroReport 17, 1747-1751. https://doi.org/10.1097/01. wnr.0000239960.98813.50. 